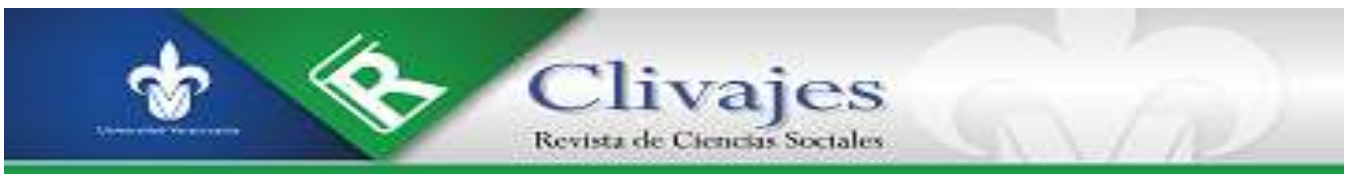

Nemesio Castillo, Esteban Eugenio Esquivel y Rogelio Rodríguez JÓVENES UNIVERSITARIOS Y NUEVAS MASCULINIDADES

Clivajes. Revista de Ciencias Sociales. Año VI, número 12, julio-diciembre 2019, pp. 108-124.

http://clivajes.uv.mx/index.php/Clivajes/editor/proofGalley/2583/4451

Instituto de Investigaciones Histórico-Sociales, Universidad Veracruzana

Clivajes. Revista de Ciencias Sociales/ISSN: 2395-9495/IIH-S, UV/Xalapa, Veracruz, México.

Recibido: 03/09/2019

Aceptado: 08/10/2019

Dictaminado: 08/II/19

Publicado: 15/11/2019

Clivajes. Revista de Ciencias Sociales (ISSN: 2395-9495), Año VI, Núm. 12, julio-diciembre, 2019 


\title{
JÓVENES UNIVERSITARIOS Y NUEVAS MASCULINIDADES
}

\author{
Nemesio Castillo Viveros ${ }^{*}$ \\ Esteban Eugenio Esquivel Santoveña** \\ Rogelio Rodríguez Hernández ${ }^{* * *}$
}

\begin{abstract}
Resumen
Este artículo da cuenta de un estudio sobre las diferencias de comportamiento de padres e hijos en cuanto al uso de la violencia en sus relaciones de pareja. Durante la investigación, 164 estudiantes universitarios hombres proporcionaron sus experiencias de perpetración de violencia hacia su pareja, así como las experiencias de violencia de sus padres hacia sus parejas, mediante la Escala de Tácticas de conflicto entre padres (CTS-between parents) y en relaciones de pareja (CTS2-versión breve). Los resultados indican una disminución en la agresión psicológica y la violencia física de los hijos contra sus parejas, en comparación con la violencia ejercida por sus padres hacia sus parejas. Este hallazgo sugiere que enfrentamos nuevas formas de masculinidad, con diferencias estadísticas importantes, entre la generación X (padres) y la de los millennials o (hijos), a la luz de los avances tecnológicos, el acceso a la información y la conciencia social acerca de los derechos humanos y de la mujer.
\end{abstract}

Palabras clave: Masculinidad, Millennials, Violencia y noviazgo

\section{INTRODUCCIÓN}

Estudiar la forma como se educa a los hombres permite generar pistas para futuras intervenciones sociales. Los estudios de género, como los estudios sobre la mujer, establecen que a hombres y mujeres se les educa de manera diferenciada. Estas diferencias, que en procesos de socialización permiten a los hombres (en comparación con las mujeres)

\footnotetext{
* Sociólogo (Universidad Veracruzana, UV), Maestro en Ciencias Sociales para el Diseño de Políticas Públicas y Doctor en Ciencias Sociales (Universidad Autónoma de Ciudad Juárez, UACJ), ha sido coordinador de la Licenciatura en Trabajo Social de la UACJ (2013-2015) y del Programa de Maestría Trabajo Social (2015-2018). Actualmente es profesor-investigador de tiempo completo en la UACJ y miembro del Sistema Nacional de Investigadores, Nivel I. Líneas de investigación: intervención social, política social y evaluación de proyectos sociales.

** Psicólogo (Universidad Autónoma del Estado de México, UAEMEX), con doctorado en Psicología (Universidad de Birmingham, Inglaterra) y posdoctorado en la misma disciplina (UAEMEX), es miembro del Comité Editorial del Journal of Aggression, Maltreatment \& Trauma (Taylor \& Francis) y de la revista Partner Abuse (Springer Publishing), y revisor de proyectos de investigación en convocatorias internas de la Universidad Iberoamericana. Candidato a Investigador nacional. Líneas de investigación: violencia, agresión, tipologías de ofensores. Profesor de Tiempo Completo en pregrado y posgrado del Departamento de Ciencias Sociales en la UACJ. Cuenta con publicaciones de circulación nacional e internacional.

*** Psicólogo, tiene estudios de Maestría en Psicología Social y Doctorado en Trabajo Social y Políticas de Bienestar Social (Universidad Autónoma de Nuevo León, UANL). Es profesor-investigador del Departamento de Ciencias Sociales de la Universidad Autónoma de Ciudad Juárez (UACJ). Es miembro del Sistema Nacional de Investigadores, Nivel 1. Líneas de investigación: violencia de pareja, sistemas de apoyo formales e informales en víctimas de la violencia de pareja, evaluación de proyectos sociales.
} 
desviarse de las normas sociales, han sido identificadas por académicos bajo la perspectiva o teoría del control (Hagan, Gillis, \& Simpson, 2016; Hagan, Simpson, \& Gillis, 1987). Desde la cultura se establecen roles/diferencias muy claros entre hombres y mujeres, y lo femenino y lo masculino.

Hay familias que intentan borrar esas diferencias y educan a sus hijos en una perspectiva no de roles de género, sino de equidad e igualdad (Díaz-Loving, Cruz-Torres, Armenta-Huarte, \& Reyes-Ruíz, 2018; Fauchier \& Straus, 2007). Parte de esta tendencia se ha sugerido a partir de los avances en derechos humanos y de la mujer; en México se reflejan particularmente en la autopercepción de mujeres y hombres en términos de normas sociales más emancipadoras y liberales, en comparación con reportes de autoconcepto que datan de mediados del siglo pasado (Díaz-Loving et al., 2015). Esta tendencia se refleja en la auto-percepción de jóvenes mexicanos, como parte de una sociedad más igualitaria que antes, y con más rasgos de individualismo social que antes (Díaz-Loving et al., 2018).

Aunados a los efectos en las estructuras familiares o dinámicas de pareja sugeridos por los cambios sociales, estudios académicos en torno a la familia han señalado el peso de la transmisión intergeneracional de la violencia derivada de experiencias previas, sea por haber presenciado dichas dinámicas o por haber sido víctima directa, es decir, por haber sufrido violencia en un contexto de maltrato o a causa de la disciplina parental. Esta transmisión de pautas de relación violenta entre individuos se presenta mediante el aprendizaje social y recientemente se ha adjudicado también a un componente neurobiológico (Haller, 2014).

\section{CARACTERÍSTICAS GENERACIONALES}

Pocos estudios aportan pistas sobre las diferencias puntuales entre generaciones, incluso acerca de si es metodológicamente pertinente y viable clasificar a las generaciones. Creemos que tener acceso a más información, la existencia de un nuevo modelo educativo y la creación de redes sociales virtuales han propiciado un cambio en el comportamiento de las personas y en su forma de interactuar con el mundo y con los otros. Para mayor precisión en el estudio de las diferencias entre generaciones, a continuación describimos algunas características de las generaciones $X$ y $Y$, esta última también conocida como la de los millennials.

De acuerdo con Vela-Valldecabres (2010), la generación $X$ está conformada por “jóvenes consumistas y conformistas que nacieron entre 1965 y 1975” (p. 370); sus edades 
rondan entre los 34 y 54 años. La generación $Y$ o millennial, por su parte, agrupa a los jóvenes nacidos entre 1983 y 1999, es decir que tendrían entre 20 y 36 años, mientras que los de la generación $Z$, conocidos como jóvenes pantalla nacieron entre 2000 y 2018, y por tanto tienen entre 1 y 19 años. Para Silvestre \& Cruz (2016): "los millennials o generación Y se pueden considerar como la población más importante de estudiantes universitarios. La mitad de esta generación está actualmente en la universidad y otra cuarta parte entrará en ella muy pronto" ( p. 473).

Varios estudios pioneros en las ciencias sociales plantean diferencias importantes entre dichas generaciones. Según Feixa (2000), la generación $Y$, a la cual también llama generación@(arroba), hay:

Tres tendencias de cambio que intervienen en este proceso: en primer lugar, el acceso universal - aunque no necesariamente general- a las nuevas tecnologías de la información y de la comunicación; en segundo lugar, la erosión de las fronteras tradicionales entre los sexos y los géneros; y en tercer lugar, el proceso de globalización cultural que conlleva necesariamente nuevas formas de exclusión social (p. 77).

Quien popularizó el término "generación X” fue Coupland (cit. en Portillo, 2012), quien hacía referencia a "una juventud marcada por las incertidumbres y las paradojas de la sociedad posmoderna, y por la falta de un sistema de valores sólido” (p. 140). Pensaba que "la asimetría en los procesos de incorporación a la tecnología de los jóvenes latinoamericanos [daba] como resultado generaciones atemporales o desfasadas en el tiempo" (p. 141).

Las prácticas educativas y de cuidado infantil han cambiado debido a la promoción de los derechos del niño; las escuelas han reformado sus modelos educativos y las familias se han adaptado a nuevas formas de cuidar y educar a los niños (un ejemplo de ello son las guarderías). La generación $Y$ ha tenido mayor contacto en la escuela, donde ha aprendido sus derechos y en esa medida ha tratado de exigirlos en su hogar.

De acuerdo con González-Pérez \& Mercado (2014), los jóvenes pertenecientes a la generación $Y$, "han crecido siendo observados, supervisados y documentados toda su vida, de hecho, de esta generación existe mucha más información que de cualquier otra. Los 'Y' toman mejores ventajas de las oportunidades que los adultos les ofrecen y son conscientes de los derechos que para ellos se han incluido en las políticas públicas” ( p. 7).

El acceso a la tecnología ha permitido que las nuevas generaciones impongan cambios con respecto a sus antecesores. Ahora, el contacto con la información de manera expedita auspicia que las dudas se puedan resolver en poco tiempo. Otro de los aspectos a 
destacar es que en las escuelas promueven la equidad de género. Actualmente, la generación de los $Y$ :

Es la más grande cohorte demográfica después de los baby boomers. Son el grupo poblacional que ha tenido mayor acceso a educación en la historia y mayores posibilidades de consumo. Crecieron con consciencia de los daños ambientales y los efectos de la discriminación en los ámbitos sociales y laborales. Y, además, siempre han convivido con las nuevas tecnologías de la información (Gonzalez-Perez \& Mercado, 2014, p. 8).

Por otro lado, los patrones de consumo se han vuelto efímeros, hacen que el apego a determinadas mercancías deje de ser un fetiche. En este sentido, podemos decir que hay un mayor distanciamiento de la cultura y las costumbres. Estamos ante un proceso de cambio fundamental de patrones de género. Se sugiere que los hombres poseen una visión distinta sobre la cultura y la tradición, tomando en cuenta que los contextos urbanos cuentan con mayor acceso a la información, lo que puede provocar un distanciamiento de los patrones o prácticas culturales esperados sobre el comportamiento masculino de los hombres, una tendencia que está en línea con otras, generales, de equidad-jerarquía e individualismo-colectivismo en México, tal como lo han documentado estudios etnopsicológicos recientes (Díaz-Loving et al., 2018). Ahora las posibilidades de sanción social mediante el uso de Facebook o, en general, de las redes sociales, pueden incidir sobre o censurar el comportamiento de los hombres y las mujeres, incluso hay patrones de comportamiento que pueden ser copiados por otros.

En cuanto a la generación $X$, ésta ha construido una nueva cultura y nuevas prácticas sociales. La idea de los roles de género no es tan precisa debido a la coexistencia de premisas psicohistórico-socioculturales tradicionales, con nuevas y más liberales creencias en dirección a la equidad y emancipación de la mujer (Díaz-Loving et al., 2015).

Según Portillo (2012): "la generación $X$ arribaba a su juventud cuando surgía internet en la segunda mitad de los noventa, mientras que la generación@ recién nacía en esos años". Los miembros de esta generación, como decíamos, también conocida como $Y$, "se han constituido en los últimos años como el actor juvenil de mayor visibilización y articulación pública”. Se han vuelto incendiarios y revolucionaros a través de las redes sociales, si bien estamos ante un "proceso social en el cual se despliegan prácticas y significados culturales que expresan las dinámicas de cambio y continuidad en un lugar y momento temporal específico".

A los jóvenes integrantes de esta generación $Y$, se le ha calificado como "malcriados", "egoístas", "impacientes"; con una educación de calidad, se adaptan a los 
cambios y prefieren un trabajo que "les llene", en lugar de un salario considerable. Son críticos y comparten casi todo. El de ellos es el mundo de la Wikipedia. La tecnología satisface su vida cotidiana. De acuerdo con el Centro de Investigaciones Pew (2015), el 83\% de los millennials duerme con su teléfono inteligente al lado de la cama” (Silvestre \& Cruz, 2016) y según Portillo, estamos ante una nueva configuración del espacio y el tiempo: "la política de los adultos espacializa en un solo lugar y tiempo, la política cultural de los jóvenes la escenifica en la cotidianeidad y en diferentes momentos”. Esta es una nueva configuración cultural en cuanto a cómo se negocia la resolución de los conflictos.

Hay un cambio generacional importante en las prácticas de resolución de conflictos, de crianza y de la autopercepción del individuo y la familia/pareja. Uno de los supuestos es que los hijos se comportan de manera distinta a sus padres. El acceso a la información permite que los jóvenes estén más informados y, por lo tanto, cambien sus patrones de comportamiento. Las redes sociales también sirven como una sanción colectiva, es decir, con cierta mesura, por ejemplo, a mostrar imágenes o comportamientos violento, esto es: no sólo los amigos revisan la información, sino también los familiares están al pendiente de lo que los unos y otros comparten. Hasta hace poco, la sanción ocurría a través de la palabra; actualmente se concreta mediante las redes sociales, donde todos pueden opinar sobre el comportamiento negativo y se sugiere una menor incidencia de comportamientos violentos en el contexto familiar.

\section{LA MASCULINIDAD Y SUS IMPLICACIONES}

Actualmente, las generaciones enfrentan un cambio de patrones para la resolución de conflictos, la crianza y la autopercepción, lo que puede traer consigo una disyuntiva para los hombres, en particular con respecto al concepto de masculinidad, un concepto central para explicar dinámicas violentas de pareja en jóvenes mexicanos desde la perspectiva de género (Fernández-Chagoya \& Ayllón-González, 2014). Si bien “masculinidad” es un concepto nuevo, hay ya una diversidad de estudios sobre el tema; es importante reflexionar y establecer algunos criterios sobre ésta.

En principio, debemos referir el concepto de género: "una categoría dinámica, construida socialmente, que tiene como base las diferencias sexuales biológicas" (Hardy \& Jiménez, 2001, p. 79). Restringir una parte del género a la biología deja algunas dudas importantes, pues no hay nada que determine los criterios que serán normados para el hombre o la mujer. Si se habla de fuerza física, hay posibilidades de que algunos hombres queden en desventaja en relación con las mujeres, ya que, con entrenamiento, éstas 
pueden desarrollarla. Debemos dejar a un lado los esencialismos, porque nos llevan estereotipar y a crear prejuicios sobre hombres y mujeres. Donde hay una mayor claridad es en el concepto de sexo:

Las personas nacen con un sexo biológico y éste acaba determinando la forma como serán tratadas socialmente por los padres, la familia y por la comunidad a la que pertenecen, para llegar a ser hombres y mujeres con atributos aceptados socialmente. Este proceso varía de una sociedad a otra y también de acuerdo con el tiempo histórico en que estas personas están insertas (Hardy \& Jiménez, 2001, p. 78).

La cultura obedece a la tradición y a las costumbres de la masculinidad. De acuerdo con Hardy y Jiménez (2001):

La construcción de la masculinidad de un nuevo ser puede comenzar cuando la pareja planifica un embarazo o cuando la mujer descubre que está embarazada. Los futuros padres empiezan a imaginarse las características que tendrá el hijo, incluyendo su sexo. Según se imaginen un niño o una niña, los padres tendrán un comportamiento diferente que comenzaría antes del parto, incluyendo la preparación del ajuar (p. 79).

Hay que considerar que este tipo de discurso concuerda con prácticas interiorizadas en los sujetos; sin embargo, las realidades de éstos no siempre se someten a estas prácticas. Nuevas generaciones de parejas se centran en el ejercicio de la igualdad y no de lo femenino o lo masculino. No es posible generalizar en la cultura, se debe pensar en la diversidad.

Es importante considerar que algunas grandes instituciones sociales funcionan como polos de influencia: "la familia, la escuela, los medios de comunicación y la sociedad en general le enseñan explícita e implícitamente la forma en que debe pensar, sentir y actuar como "hombre" (Hardy \& Jiménez, 2001). Y es evidente que para conseguir que el sujeto tome consciencia y se distancie de estas grandes instituciones debe pasar por procesos de reflexión y critica.

Cabe destacar, además, que culturalmente la masculinidad se asocia con factores reconocidos por la sociedad: el hombre "posee un elemento clave que es el poder. Ser hombre significa tener y ejercer poder. El poder asociado a la masculinidad exige poseer algunas características, tales como ganar, ordenar, lograr objetivos y ser duro" (Hardy \& Jiménez, 2001).

Por supuesto, lo femenino o lo masculino no están presentes en el hombre o la mujer por el hecho de serlo; cada sexo desarrolla elementos de apropiación, conforme a lo que socialmente se reconoce y/o premia, de tal modo que las características tradicionales 
de hombres y mujeres son evidentes en cómo los mexicanos se han autopercibido (Díaz Loving, Rivera Aragón, Villanueva Orozco \& Cruz Martínez, 2011).

\section{EDUCACIÓN EN HOMBRES Y CAMBIOS CULTURALES}

La teoría feminista ha derribado muchas de las estructuras de poder que durante muchos años construyeron los hombres, y trabaja para dilucidar las estructuras de desigualdad entre hombres y mujeres. Es su caso, los estudios sobre masculinidad tratan de entender la construcción social del hombre para edificar una sociedad más justa e igualitaria.

Actualmente, la división social del trabajo entre hombres y mujeres tiene mayor complejidad; hombres y mujeres desempeñan trabajos semejantes, aunque algunos oficios tienen mayor presencia de hombres, como la albañilería; sin embargo, muchas mujeres realizan también ese trabajo. Hay que decir, además, que se ha avanzado en relación con el cuidado de los hijos; ahora esta actividad es generalmente compartida, al igual que las labores domésticas. De acuerdo con Guevara (2008):

Las nuevas formas de participación social y de autonomía de las mujeres han puesto a la masculinidad en crisis, y se ha abierto un debate sobre diversas tesis acerca de los hombres, entre ellas: si han cambiado o no; si ya participan en el trabajo doméstico; si su identidad está todavía en ser proveedores; si ejercen una paternidad menos autoritaria; si ahora se encaminan por la ruta de "nuevas masculinidades", preguntas que inevitablemente nos conducen de nuevo a los individuos y que al enfocarse sólo en los atributos personales pierden de vista el carácter estructurado y estructurante del orden de género y olvidan que las posibilidades de poder de los hombres derivan de la posición social que ocupan y que les otorga oportunidades que no tendrían si ocuparan una distinta (p. 73).

En la década de 1970, comenzaron a aparecer los “Men’s Studies; su particularidad consiste en dejar de lado al hombre como representante general de la humanidad y adoptar el estudio de la masculinidad y las experiencias de los hombres como específicas de cada formación socio-histórico-cultural" (Minello, 2002, p. 12). El término "masculinidad" tiene muchas variantes; de acuerdo con Martini (2002, p. 719), puede ser considerado:

i) un atributo personal que los distintos hombres poseen en distintas magnitudes;

ii) un rasgo de personalidad, que puede ser más o menos permanente en cada individuo;

iii) una esencia inscrita en la naturaleza de los varones;

iv) un papel en la organización social (proveedor, protector, etc.);

v) todo lo que hacen o piensan los hombres;

vi) todo aquello que hagan o piensen en función de ser hombres;

vii) lo que hacen o piensan algunos varones, considerados paradigmáticos;

viii) dentro de las relaciones de género". 
Los estudios sobre las mujeres, las masculinidades o de género han permeado en la estructura social, lo cual ha propiciado que "muchos varones que se han dedicado a esta tarea comparten con las mujeres el interés en una sociedad más segura y más justa. Están interesados también en eliminar la discriminación en todas sus formas e, incluso, en promover una economía menos jerárquica y más incluyente” (Guevara, 2008). Incluso a determinadas palabras se les ha atribuido un carácter peyorativo. Para Martini (2002): Machismo es un nombre aplicado a situaciones contradictorias. Su uso, más que aportar nuevos
conocimientos sobre la masculinidad, confunde; cada lector o lectora puede darle un significado
distinto y ofrece una falsa sensación de saber. Por otra parte, contribuye también a una reificación
o quizás esencialización del sujeto, al declararlo macho, no reconocerle ambigüedades o
contradicciones en sí mismo, negarle la riqueza de una identidad construida por muchos factores y
condenarlo a un estereotipo (p. 722 ).

El concepto "machista" tiene una connotación peyorativa en la sociedad; eso contribuye a que los hombres nieguen el apelativo y adopten un criterio de equidad e igualdad en sus relaciones con hombres y mujeres. Así pues, para Martini (2002), el "machismo parece una palabra sólo para ser hablada; su significado depende de la entonación y de la expresión de quien la dice, quizá del contexto. Si se mantuviera en el lenguaje verbal, no habría más que tener un oído atento. Pero no sucede lo mismo cuando se traslada a la escritura y, más aún, se le convierte en una categoría de análisis” (p. 721). Cabe señalar que hay otras áreas por explorar. No es lo mismo hablar del hombre occidental que del hombre latinoamericano, con su herencia desde la época precolonial; la masculinidad, como se estudia ahora, tiene "una huella occidental” (p. 719).

En este contexto de cambios intergeneracionales, el presente estudio se plantea como objetivo examinar los patrones de uso de la violencia, por padres e hijos, con el fin de dirimir conflictos en sus relaciones de pareja. La hipótesis aquí presentada parte del cambio intergeneracional (entre los padres y los hijos) en cuanto al usos de la violencia física para dirimir sus conflictos o diferencias de pareja. El enfoque de este estudio, en la generación $X$ y los millennials ( $Y$ ), se asocia con la noción de que los hijos (los millennials) tienen mayor acceso a la información y que en su educación formal han recibido más datos sobre derechos humanos y de la mujer (enfoque de género), además de su autopercepción, a la luz de los cambios sociales y avances precisamente en derechos humanos y de la mujer.

Este estudio es de carácter descriptivo y exploratorio. Tiene la intención de encontrar algunas pistas sobre patrones conductuales que pudieran apuntar hacia nuevas masculinidades. El propósito de este tipo de investigación es reflexionar sobre la necesidad 
de una realizar una intervención social con hombres, pues cuando se habla de violencia ejercida contra la mujer, a ésta se le atiende en términos psicológicos, legales, económicos, etc., mientras que para el hombre se piensa más en castigos punitivos, pero no se atiende su comportamiento. Este objetivo se abordó, específicamente, a través de la siguiente pregunta de investigación: ¿Cuál es la diferencia entre los padres y los hijos en el uso de la violencia en pareja?

Con el interés de precisar las diferencias entre los padres y los hijos, aquí se presentan algunas preguntas por separado. Nuestra hipótesis plantea que hay un cambio de generación entre los padres y los hijos en cuanto al uso de la violencia física para dirimir sus conflictos $o$ diferencias de pareja. Nos concentramos en la generación $X$ y la Millennial ( $Y$ ), pues consideramos que los hijos tienen mayor acceso a la información, y debido a su educación formal, existe en ellos mayor consciencia sobre derechos humanos y el enfoque de género.

\section{MÉTODOLOGÍA}

Se realiza una investigación social en el campo de los estudios de género, con enfoque cualitativo, el cual suele tener mayor aceptación y tradición en la comunidad académica, aunque los enfoques cuantitativos también tienen algo que decir. Las bases de datos y las encuestas sirven para entender el comportamiento de la población con mayores posibilidades de generalización. Tal como afirma Minello (2002), hay un uso adecuado y profundo de las técnicas cualitativas para el estudio de masculinidades; sin embargo:

por una errónea, aunque difundida visión de una oposición teórico-metodológica entre métodos cualitativos y cuantitativos, no se aprovechan los datos censales o de encuesta que, utilizados con las precauciones debidas, pueden dar perfiles globales de la masculinidad. Tampoco son muy aprovechados los documentos, que pueden ser periódicos o revistas, testamentos, juicios de divorcio, juicios penales por violencia (acoso sexual, violación, violencia doméstica, etc.), incesto, juicios de trabajo u otros, procesos eclesiásticos, manuales de confesión, así como informes institucionales (p. 27).

Esta investigación utiliza también un enfoque cuantitativo y forma parte de un proyecto internacional que examina aspectos de parentalidad, experiencias de violencia en la familia de origen y su relación con experiencias de violencia en el noviazgo e indicadores asociados de salud mental en jóvenes universitarios. 


\section{Muestra}

En el estudio participaron 397 estudiantes de ambos sexos (63.5\% mujeres), que actualmente cursan estudios de licenciatura en ciencias sociales, ciencias administrativas e ingenierías en la Universidad Autónoma de Ciudad Juárez (UACJ), México. Los criterios de inclusión para colaborar en la investigación fueron los siguientes: 1) que los jóvenes estuvieran o hubiesen estado en una relación de pareja; 2) que dicha relación hubiese tenido por lo menos un mes de duración, y 3) que la edad de los jóvenes estuviese comprendida entre los 17 y 29 años. Se excluyó a los estudiantes que jamás habían tenido una relación de pareja y a los casados o que dijeron vivir en cohabitación. Para este artículo únicamente se trabajó estadísticamente con los hombres, es decir, con 164 casos.

\section{Instrumentos}

Escala de violencia/maltrato entre padres (versión corta CTS-between parents; Straus \& Douglas, 2004; Straus, Hamby, Boney-McCoy, \& Sugarman, 1996): Se trata de una escala de cuatro ítems que evalúan comportamientos controladores (agresión psicológica; e.g., "mi padre insultó o gritó a mi madre”; “mi padre destrozó algo que pertenecía a mi madre o amenazó con pegar a mi madre?") y violentos (e.g., "mi padre empujó o abofeteó a mi madre”; “¿mi padre dio un puñetazo, una patada o una paliza a mi madre?”) entre ambos miembros de la pareja, como resultado de algunas formas violentas de resolver argumentos o puntos de vista que se originan del conflicto de interés, inherente a cualquier relación de pareja, y/o motivaciones subyacentes a dichos intereses. El índice alfa de confiabilidad para la escala de perpetración de violencia del padre hacia la madre en el presente estudio es de $\alpha=.83$.

Escala de violencia en el noviazgo (versión corta CTS2; Straus \& Douglas, 2004; Straus et al., 1996): Al igual que la versión de violencia entre padres, esta escala presenta comportamientos indicativos de agresión psicológica (e.g., "insulté o grité a mi pareja"; "destrocé algo que pertenecía a mi pareja o amenacé a mi pareja con golpearla") y violencia física (e.g., "empujé o le di una bofetada a mi pareja, golpeé, pateé o le di una paliza a mi pareja”), existentes en una relación de pareja o noviazgo. Las escalas CTS2 cuentan con evidencia de validez y confiabilidad demostrada en estudios empíricos internacionalmente y en México (Straus, 2007). El índice de confiabilidad para la escala de perpetración de la violencia en el presente estudio es de $\alpha=.75$. 


\section{Procedimiento}

La aplicación del cuestionario de autoreporte se llevó a cabo en las aulas al finalizar las clases y en promedio tuvo una duración aproximada de una hora y media. La participación en el estudio fue voluntaria y anónima, mientras que el consentimiento informado lo otorgaron los estudiantes al contestar el cuestionario. Previamente a la recolección de la información, se obtuvo el dictamen aprobatorio por parte del Comité de Ética y Bioética de la UACJ.

Análisis de resultados

Los patrones de género están presentes en la generación $X$. De acuerdo con la encuesta aplicada a los jóvenes universitarios, el 45.9\% afirmó que la madre tenía más responsabilidad que el padre en cuanto a la disciplina correctiva de los hijos; el 14.8\% dijo que su madre era un poco más responsable que su padre en cuanto a la disciplina correctiva, y el 32\% mencionó que sus padres compartían la responsabilidad; tan solo el 4.4\% dijo que su padre era un poco más responsable que su madre en la disciplina, y para el 2.6\%, su padre tenía mucha más responsabilidad al respecto.

En cuanto a la violencia ejercida por el padre sobre la madre, con insultos y gritos (agresión verbal o psicológica), más de la mitad de los participantes afirmaron que dichas experiencias se presentaron en distintos niveles de cronicidad (números de veces) durante un año, cuando ellos tenían 10 años de edad (Tabla 1).

Tabla 1.

Experiencias de agresión psicológica (insultos o gritos) del padre hacia la madre

\begin{tabular}{lc}
\hline Cronicidad con la que ocurrieron las agresiones & $\%$ \\
\hline Nunca ha sucedido & 44.8 \\
No sucedió aquel año, pero sucedió antes o después & 12.5 \\
Una vez aquel año & 8.9 \\
Dos veces aquel año & 8.9 \\
3 a 5 veces aquel año & 10.9 \\
6 a10 veces aquel año & 4.7 \\
11 a 20 veces aquel año & 3.6 \\
Más de 20 veces aquel año & 3.1 \\
\hline Nota: Elaboración propia con datos de la Encuesta. Los resultados no totalizan 100\% debido a casos faltantes. \\
En cuanto a la violencia física con empujones o bofeteadas del padre a la madre, un 23.3\% de los participantes reportó \\
que sus padres la ejercieron (Tabla 2).
\end{tabular}


Tabla 2.

Experiencias de violencia física (empujones o bofetadas) del padre hacia la madre

\begin{tabular}{lc}
\hline Cronicidad con la que ocurrieron las agresiones & $\%$ \\
\hline Nunca ha sucedido & 73.4 \\
No sucedió aquel año, pero sucedió antes o después & 12.5 \\
Una vez aquel año & 2.6 \\
Dos veces aquel año & 3.1 \\
3 a 5 veces aquel año & 1.0 \\
6 a 10 veces aquel año & 1.0 \\
11 a 20 veces aquel año & 2.6 \\
Más de 20 veces aquel año & .5 \\
\hline Nota: Elaboración propia con datos de la Encuesta. Los resultados no totalizan 100\% debido a casos faltantes.
\end{tabular}

Por cuanto respecta a los reportes de hombres jóvenes en relación con el trato que le dan a su novia, se encontró que un mayor porcentaje (en relación a sus padres) nunca ejerció agresión psicológica (Tabla 3).

Tabla 3.

Experiencias de agresión psicológica (insultos o gritos) de los hijos hacia la pareja (mujer)

\begin{tabular}{lc}
\hline Cronicidad con la que ocurrieron las agresiones & $\%$ \\
\hline Nunca ha sucedido & 56.8 \\
No sucedió aquel año, pero sucedió antes o después & 14.6 \\
Una vez aquel año & 7.3 \\
Dos veces aquel año & 4.7 \\
3 a 5 veces aquel año & 2.1 \\
6 a 10 veces aquel año & 1.0 \\
11 a 20 veces aquel año & 1.0 \\
Más de 20 veces aquel año & .5 \\
\hline Nota: Elaboración propia de la tabla con datos de la encuesta. Los resultados no totalizan un 100\% debido a casos faltantes.
\end{tabular}

En relación con la violencia física del hombre universitario contra su pareja, se encontró que un $14.6 \%$ ejerció esta violencia, siendo una pequeña proporción la que lo reportó con un alto nivel de cronicidad (Tabla 4).

Tabla 4.

Experiencias de violencia física (empujones o bofetadas) de los hijos hacia la pareja (mujer)

\begin{tabular}{lc}
\hline Cronicidad con la que ocurrieron las agresiones & $\%$ \\
\hline Nunca ha sucedido & 71.9 \\
No sucedió aquel año, pero sucedió antes o después & 8.9 \\
Una vez aquel año & 3.1 \\
Dos veces aquel año & 1.6 \\
Más de 20 veces aquel año & 1.0 \\
\hline
\end{tabular}

Nota: Elaboración propia de la tabla con datos de la encuesta. Los resultados no totalizan un 100\% debido a casos faltantes. 
En relación con los niveles de agresión psicológica, se encontró que los padres ejercían mayores niveles $(M=1.72, D E=2.038)$ de agresividad contra la madre, en comparación con la agresión ejercida por los hijos hacia la pareja $(M=0.78, D E=$ 1.354; $t[164]=5.662, p=0.001)$. Esto refleja que hay un cambio significativo entre los patrones de comportamiento de los padres a los hijos, es decir, los millennials ejercen menos violencia psicológica que la generación $X$.

Con respecto a las experiencias de violencia física, se encontró que los padres la ejercían hacia la madre $(M=0.59, D E=1.359)$ con mayores niveles, en comparación con la ejercida por los hijos hacia su pareja $(M=0.30, D E=0.940 ; t[164]=2.568, p$ $=0.001$ ), lo cual evidencia que hay un cambio significativo entre los patrones de comportamiento de los padres a los hijos, es decir, los millennials son físicamente menos violentos que la generación $X$.

Asimismo, en lo que refiere a las escalas de violencia total (agresión psicológica y violencia física), se encontró que los padres perpetraron mayores niveles de violencia hacia sus parejas $(M=3.1688, D E=4.69071)$, en comparación con la perpetrada por los hijos jóvenes hacia sus parejas $(M=1.5063, D E=2.82842$; $\mathrm{t}[159]=4.902, p=$ 0.001), lo cual refleja que hay un cambio significativo entre los patrones de comportamiento de los padres a los hijos, es decir, los millennials son menos violentos que la generación $X$ (Tabla 3).

\section{DiSCUSIÓN}

Resumiendo lo encontrado en el presente estudio, hubo diferencias entre los padres y los hijos. Los resultados señalan que hay un cambio generacional importante en relación con las conductas violentas perpetradas hacia la pareja. Cabe señalar que se habla de jóvenes mexicanos universitarios, cuyas redes sociales, prácticas de convivencia, su percepción de género y las nuevas formas de asumir la masculinidad son fundamentales.

Los hallazgos son congruentes con el cambio generacional sugerido, a raíz de los efectos de los avances tecnológicos y el acceso a la información, con una mayor conciencia acerca de los derechos humanos y de la mujer entre los jóvenes mexicanos, así como con los cambios en su autopercepción en relación con los actuales roles de género y la tendencia del joven mexicano a percibirse en una sociedad y cultura con más rasgos individualistas y más cercanas a la equidad que a las estructuras jerárquicas de género, que en el pasado (Díaz-Loving et al., 2018). 
Actualmente existe, además, una gran cantidad de organizaciones e instituciones que promueven una vida libre de violencia entre hombres y mujeres. Como afirma Corleto Buquet (2011): "hay una gran cantidad de grupos de la sociedad civil, organizados de diferentes maneras, que han impulsado la incorporación de esta perspectiva en distintos temas sociales, logrando grandes avances en las esferas de la salud y los derechos sexuales y reproductivos, en los temas de violencia y discriminación, entre otros” (p. 212). Ejemplo del reconocimiento del reto del hombre ante estas nuevas masculinidades son los cambios en la Ley General de Acceso de las Mujeres a una Vida Libre de Violencia, en cuanto atañe a la gestión de recursos (Cámara de Diputados, 2011), para la conducción de programas orientados a ofensores de violencia de pareja ya existentes en algunas entidades de México (Esquivel-Santoveña \& Da Silva, 2016).

Una de las críticas hacia esta tendencia de cambio en patrones conductuales encontrados en jóvenes mexicanos, aduce al hecho de que se trata de jóvenes que se hallan en una relación de pareja menos consolidada y que las dinámicas en las relaciones de pareja tienden a ser distintas y asimétricas. Si bien es plausible que las dinámicas de algunas parejas sufran cambios en un sentido de mayor o menor equidad, como lo sugiere el enfoque tipológico de ofensores de violencia de pareja (Johnson, 2008), una de las fortalezas del presente estudio reside en registrar experiencias de violencia con instrumentos o escalas que han estandarizado las conductas que se indagan en padres e hijos reportados por un mismo participante.

La diversidad de instrumentos de medición utilizados en los estudios acerca de violencia de pareja ha sido señalada en la literatura académica como una de las barreras para comparar resultados provenientes de diversas fuentes (Straus, 2007). Al menos en el contexto universitario del presente estudio, hay elementos estadísticos que permiten sugerir que existe un cambio entre la generación $X$ y la Millennial. En general, las experiencias de violencia ejercida por padres e hijos hacia la pareja son consistentes con prevalencia reportada internacionalmente (Straus, 2009).

Otro de los aspectos destacados y que explican el tema es que, como señala Corleto Buquet (2011), en México se ha establecido una serie de:

centros, programas o institutos dedicados a los estudios de género en las universidades, que son, sin lugar a dudas, los terrenos de producción de nuevo conocimiento; en segundo lugar, la incorporación de estas temáticas en la formación de las y los jóvenes universitarios, o sea, el impacto de la temática de género en los programas y planes de estudio; y en tercer lugar la 
institucionalización y transversalización de la perspectiva de género en las estructuras universitarias" (p. 213).

Al parecer, si asumimos una nueva forma de vivir la masculinidad, otra forma de ser hombre, tendremos que desarrollar nuevos elementos teóricos y metodológicos para la comprensión de la realidad social. Debemos incorporar una nueva mirada a las distintas "formas de discriminación al poner de manifiesto que la condición social de desigualdad entre hombres y mujeres responde a un complejo sistema de relaciones sociales, arraigado en los significados que el orden cultural atribuye a la feminidad y a la masculinidad" (Corleto Buquet, 2011, p. 214).

En cuanto a las limitaciones del estudio, se incluye la naturaleza transversal de éste, la naturaleza de la muestra, por tratarse de jóvenes mexicanos más educados y cuya visión de familia y relaciones de pareja pudieran diferir de las opiniones de jóvenes y adultos mexicanos con un menor nivel educativo, que residen en contextos rurales con menor acceso a la información.

Uno de los aspectos a explorar en futuras investigaciones, es la adopción de un enfoque cualitativo que permita entender, en particular, las relaciones entre hombres y mujeres. También, trabajar las variables de estado civil, nivel de escolaridad, nivel socioeconómico, religión y lugar de nacimiento, lo cual podría aportar mayores elementos para encontrar patrones de comportamiento entre la generación $X$ y la Millennial. Un estudio de carácter cuantitativo que involucre tanto autopercepción de roles de género, acceso a la información en derechos humanos y experiencias de violencia en nuevas generaciones de jóvenes mexicanos, habrá de esclarecer sin duda el impacto de los cambios sociales y tecnológicos sobre cómo se perciben estos jóvenes en relación con las formas dinámicas violentas y no violentas al relacionarse en pareja.

Creemos, sin embargo, que este estudio libra uno de los obstáculos metodológicos más comúnmente señalados en la literatura en cuanto a la medición de comportamientos y los resultados son alentadores, en el sentido de que evidencian una disminución de agresiones y violencia en las relaciones de pareja.

\section{CONCLUSIONES}

Debemos reconocer que la diferencia entre la generación $X$ y la Millennial es el acceso a los diferentes recursos de información y la educación básica que recibieron sobre las áreas de justicia, derechos humanos, medio ambiente y género. Internet nos permite estar 
conectados con personas de distintos lugares que nos pueden apoyar con información o con sus experiencias; también da la posibilidad de consultar, de manera gratuita, toda una serie de documentos, como la Constitución, así como normas y reglamentos, lo que auspicia la formación de personas informadas.

Las redes sociales son otro elemento constitutivo que ha posibilitado el internet. Actualmente las personas pueden exponer sus experiencias de vida en una u otra red social, lo cual ayuda a otras a estar atentas con respecto a las diferentes problemáticas que enfrentan; por ejemplo, el movimiento social feminista llamado "Me Too" tuvo tanto impacto social por la potencialidad de las redes sociales. Lo que estamos viviendo es que las redes sociales permiten exponer asuntos privados a escala global; en otras palabras, permiten que las noticias locales alcancen importancia global. Asuntos considerados privados en las instituciones, sean públicas o privadas, con las redes sociales modifican de manera profunda la vida pública. Lo que vivimos es un colapso de lo local; con las redes sociales todo se vuelve global y ello las convierte en un mecanismo de regulación, incluso de las conductas sociales.

\section{Referencias}

CÁmara de Diputados. (2011). Ley General de acceso de las mujeres a una vida libre de violencia (General Access Law of Women to a Life Free of Violence). Cámara de Diputados. México.

Corleto Buquet. A. G. (2011). Transversalización de la perspectiva de género en la educación superior Problemas conceptuales y prácticos. Perfiles Educativos, XXXIII, pp. 211-225.

Díaz-Loving, R., Cruz-Torres, C. E., Armenta-Huarte, C., \& Reyes-Ruíz, N. E. (2018). Variations in horizontal and vertical individualism-collectivism in four regions in Mexico. International Journal of Social Psychology.

, Saldívar, A., Armenta-Hurtarte, C., Reyes, N. E., López, F., Moreno, M., Correa, F. E. (2015). Creencias y Normas en México: Una Actualización del Estudio de las Premisas Psico-Socio-Culturales. Psykhe, 24(2), pp. 1-25.

, Rivera-Aragón, S., Villanueva-Orozco, G. B. T., \& Cruz Martínez, L. M. (2011). Las premisas histórico-socioculturales de la familia mexicana: exploración desde las creencias y las normas. Revista Mexicana de Investigación En Psicología, 3(2), pp. 128-142.

Esquivel-Santoveña, E. E. \&, \& Da Silva, T. (2016). Domestic Violence Intervention Programs for Perpetrators in Latin America and the Caribbean. Partner Aubse, 7(3), 
316-352.

Fauchier, A., \& STRaus, M. A. (2007). Dimensions of discipline by fathers and mothers as recalled by university students. In International Family Violence and Child Victimization Research Conference. Portsmouth, New Hampshire: University of New Hampshire.

FEIXA, C. (2000). Generación@ la juventud en la era digital. Nómadas, 13(Octubre), pp. 75-91.

Fernández-Chagoya, M. A., \& Ayllón-GonzÁlez, R. E. (2014). Aprendiendo a querer: noviazgos libres de violencia Guía metodológica para prevenir la violencia contra las mujeres entre estudiantes de secundaria (Learning to love: Dating relationships free of violence. A methodological guide to prevent violence against women in junior high schoool). Mexico: Gendes, A. C.

GonZalez-Perez, A., \& Mercado, H. (enero-junio, 2014). Gerenciando la Generación Y o el reto Millenials. AD-Minister, 24, pp. 7-8.

GUEVARA, E. (enero-abril, 2008). La masculinidad desde una perspectiva sociológica. Una dimensión del orden de género. Sociológica, 23, pp. 71-92.

Hagan, J., Gillis, A. R., \& Simpson, J. (2016). The Class Structure of Gender and Delinquency : Toward a Power-Control Theory of Common Delinquent Behavior Author ( s ): John Hagan , A . R . Gillis and John Simpson Published by : The University of Chicago Press Stable URL: http://www.jstor.org/stable/, 90(6), pp. 1151-1178.

, Simpson, J., \& Gillis, A. R. (1987). Class in the Household : A Power-Control Theory of Gender and Delinquency Published by : The University of Chicago Press Stable URL: http://www.jstor.org/stable/2780039 Accessed : 20-03-2016 15 : 43 UTC Your use of the JSTOR archive indicates your accept, 92(4), 788-816.

HALlER, J. (2014). Neurobiological bases of abnormal aggression and violent behaviour. Neurobiological Bases of Abnormal Aggression and Violent Behaviour. London, New York: Springer.

Hardy, E., \& JimÉNEZ, L. (2001). Masculinidad y género. Revista Cubana de Salud Pública, 27(2), pp. 77-88.

JOHnSOn, M. P. (2008). A typology of domestic violence: Intimate terrorism, violent resistance and situational couple violence. Boston: Northeastern University Press.

Martini, N. (2002). Masculinidades: un concepto en construcción. Nueva Antropología, 8(61), pp. 11-30.

- (septiembre-diciembre, 2002). Notas de investigación Los estudios de 
masculinidad. Estudios Sociológicos, XX(3), pp. 715-732.

Portillo, M. ET. AL. (diciembre, 2012). De la generación X a la generación @. Trazos transicionales e identidades juveniles en América Latina. Ultima Década, 37, pp. 137-174.

Silvestre, E., \& Cruz, O. (2016). Conociendo la próxima generación de estudiantes universitarios dominicanos a través de las redes sociales. Ciencia Y Sociedad, 41(3), pp. 475-503.

Straus, M. A. (2007). Conflict Tactics Scales. Encyclopedia of Domestic Violence, pp. 190197.

. (2009). Violence between parents reported by male and female university students:

Prevalence, severity, chronicity, and mutuallity. Journal of Aggression, Conflict and Peace Research, 1(1), pp. 4-12.

, \& Douglas, E. M. (2004). A short form of the revised Conflict Tactics Scales, and typologies for severity and mutuallity. Violence and Victims, 19, 507-520.

Straus, M. A., Hamby, S. L., Boney-McCoy, S., \& Sugarman, D. B. (1996). The revised Conflict Tactics Scales (CTS2). Journal of Family Issues, 17(3), pp. 283-316.

Vela-Valldecabres, D. (2010). Prolegómenos de la Generación X. Algunas manifestaciones cinematográficas. Palabra Clave, 13(2), pp. 369-286. 